\title{
Du genre Tergestia M. Stossich 1899 (Trematoda Digenea)
}

\author{
par Robert Ph. DOLLFUS \\ Laboratoire de Zoologie (Vers), associé au C.N.R.S. ( $\mathrm{P}^{r}$ A.-G. ChaBaUd), \\ Muséum national d'Histoire naturelle, 57, rue Cuvier, F 75005 Paris
}

\section{Résumé}

Parmi les espèces du genre Tergestia, certaines ont la bifurcation intestinale en arrière de la ventouse ventrale, je les place dans le sous-genre Tergestia; d'autres l'ont en avant, je les place dans le sous-genre Theledera $=$ Cithara .

Dans quelques cas, le même nom spécifique a été employé à tort pour des spécimens ayant, les uns la bifurcation intestinale en avant de la ventouse ventrale (s.g. Theledera), les autres cette bifurcation en arrière (s.g. Tergestia).

Une nouvelle espèce est décrite: Tergestia (Tergestia) mauritanica R.Ph.D., récoltée en Mauritanie par Théodore Monod.

\section{Summary}

Some of the species of the genus Tergestia present the intestinal bifurcation behind the ventral sucker. I place these in the subgenus Tergestia. Other species present the intestinal bifurcation before the ventral sucker. I place these in the subgenus Theledera $=$ Cithara .

In some cases, the same specific name has been employed erroneously for specimens with intestinal bifurcation before the ventral sucker (s.g. Theledera) and for specimens with intestinal bifurcation behind it (s.g. Tergestia).

A new species in described: Tergestia (Tergestia) mauritanica R.Ph.D., gathering in Mauritania by Theodore Monod.

(*) Parasitologia mauritanica. Digénétiques de Téléostéens $\left(3^{\circ}\right.$ note). 
Autant que l'on peut en juger d'après la diversité morphologique des cercaires des genres classés dans la fam. Fellodistomatidae (W. Nicoll 1909) (au sujet de cette diversité, voir R. Ph. Dollfus 1964, p. 768), (1), cette famille n'apparaît pas comme homogène dans son acception actuelle; c'est pourquoi la sous-famille Tergestiinae (Skrjabin \& Kowal 1957) était bien justifiée.

Le genre Tergestia lui-même n'est pas homogène. Je propose sa division en deux sous-genres :

s.g. Tergestia (type laticollis Stossich) pour les espèces dont l'œsophage se divise en arrière de la ventouse ventrale,

s.g. Theledera Edwin Linton 1910 (genre)=Cithara G. A. Mac Callum 1917 (genre) (type Theledera pectinata (E. Linton 1905) pour les espèces dont l'œsophage se divise en avant de la ventouse ventrale.

Dans la collection des distomes récoltés en Mauritanie, les Tergestia sont représentés par environ 12 individus provenant du tube digestif de:

$1^{\circ}$ Pomatomus saltatrix (L. 1758) Lacepède $1802=$ Temnodon saltator (L. 1758) G. Cuvier 1818 (Pomatomidae). $1^{\text {re }}$ croisière du S.S. «Boula matari » décembre 1922 et $2^{\circ}$ croisière, mai 1923.

$2^{\circ}$ Lichia vadigo (A. Risso 1810) $=$ Campogramma lirio R. Ph. Dollfus 1955, nom. nov. (Carangidae). $1^{\text {re }}$ et $2^{\circ}$ croisière du S.S. "Boula Matari ».

Pour les individus récoltés chez Pomatomus, je propose l'appellation Tergestia (Tergestia) mauritanica n. sp.

Description : Corps allongé, peu épais, peu large, très atténué postérieurement, à pointe mousse. Un des plus grands individus est long de $3,3 \mathrm{~mm}$, large de $0,5 \mathrm{~mm}$. La cuticule est dépourvue d'épines. Ventouse orale à ouverture ventrale, plus longue que large, $0,23 \times 0,18 \mathrm{~mm}$, ou presque circulaire, avec un diamètre de $0,180-0,185 \mathrm{~mm}$. Elle est pourvue dorsalement et latéralement d'appendices musculaires linguiformes, au nombre d'environ 12. Il n'y a pas de plis cuticulaires transversaux au niveau du pharynx, mais, de chaque côté, 6 protubérances marginales sacciformes, dont l'extrémité libre est terminée par deux très courts filaments. Il n'y a pas de prépharynx. Le pharynx est à bords latéraux parallèles, environ deux fois plus long que large, il mesure, par exemple, 0,20 à 0,215 de long, sur 0,110 à $0,115 \mathrm{~mm}$ de large. L'œsophage est très long et plutôt large, il dépasse un peu postérieurement la ventouse ventrale; il se divise en deux caeca qui atteignent l'extrémité postérieure du corps.

La ventouse ventrale est un peu plus longue que large, par exemple $0,37 \times 0,33 \mathrm{~mm}$, ou presque circulaire $0,370 \times 0,375$; elle est située dans la moitié antérieure du corps, son bord antérieur est distant d'environ $1 \mathrm{~mm}$ de l'extrémité antérieure pour un individu long d'environ $3,3 \mathrm{~mm}$.

Les testicules sont ovales, allongés, intercaecaux, un peu en diagonale, dans la partie postérieure du corps; ils sont subégaux, l'antérieur est, chez quelques individus, un peu

(1) Sur le stade cercaire des Tergestia, voir L. M. Angel, 1960, p. 76-85 et R. Ph. Dollfus, 1964, p. 768-769. 


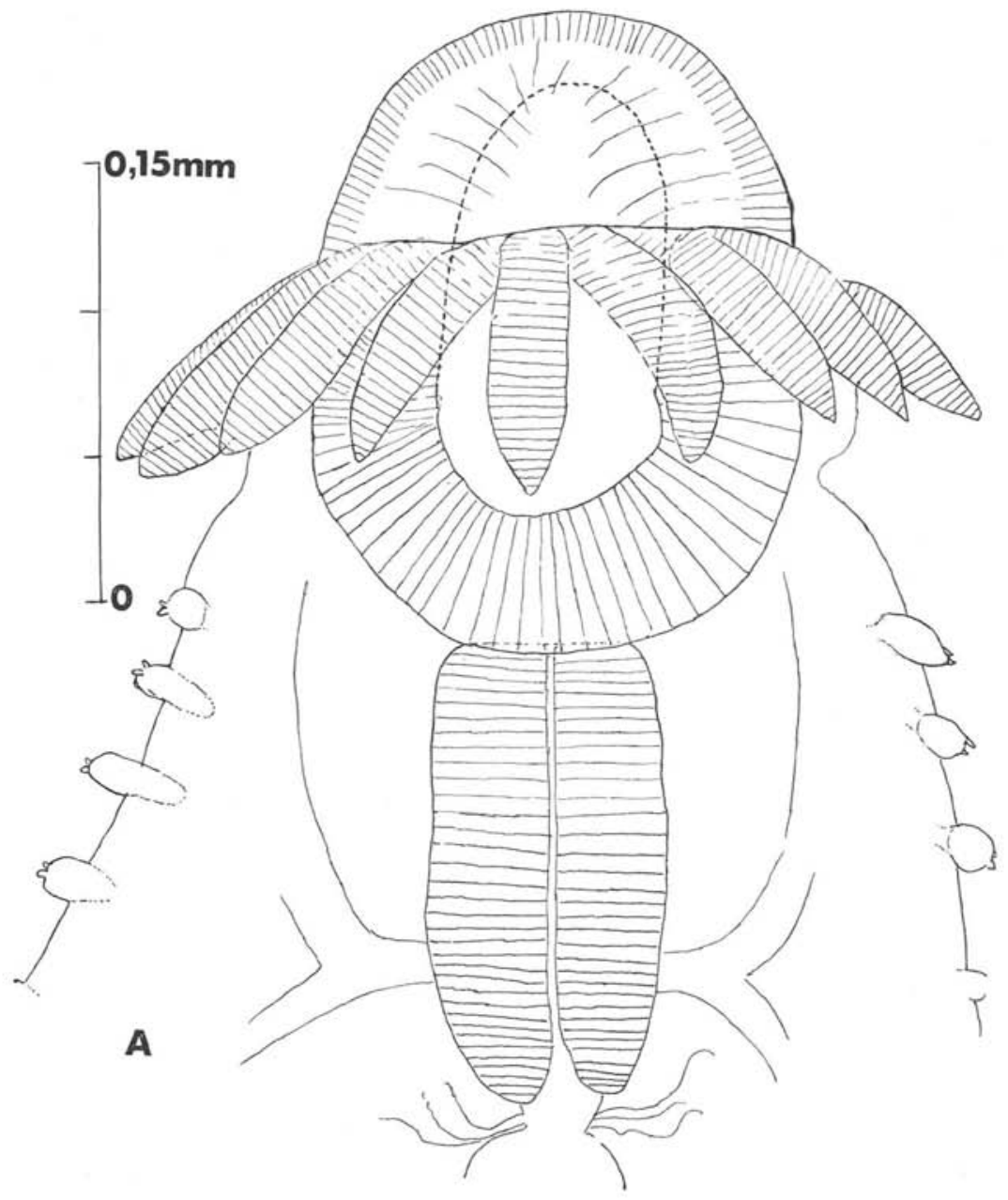

Fig. A. - Tergestia mauritanica n. sp. de Pomatomus saltatrix (L.). Région bucco-pharyngienne. Vue dorsale. Port-Etienne (Mauritanie)

plus petit que le postérieur (par exemple: testicule antérieur 0,30-0,35 $\times 0,15 \mathrm{~mm}$; testicule postérieur: $0,38 \times 0,12 \mathrm{~mm}$ ).

Poche du cirre comprenant une partie antérieure dilatée, ovale, située contre le bord antérieur de la ventouse ventrale et une partie postérieure botuliforme, très longue, mesurant par exemple 0,45-0,50 $\mathrm{mm}$ de long, avec un diamètre de 0,06-0,08 $\mathrm{mm}$, celle-ci rectiligne s'étend dorsalement à la ventouse ventrale; son extrémité antérieure est à peu près au niveau du bord antérieur de celle-ci; postérieurement, elle dépasse la ventouse, atteignant le niveau de la bifurcation intestinale; elle contient une vésicule séminale tubu- 


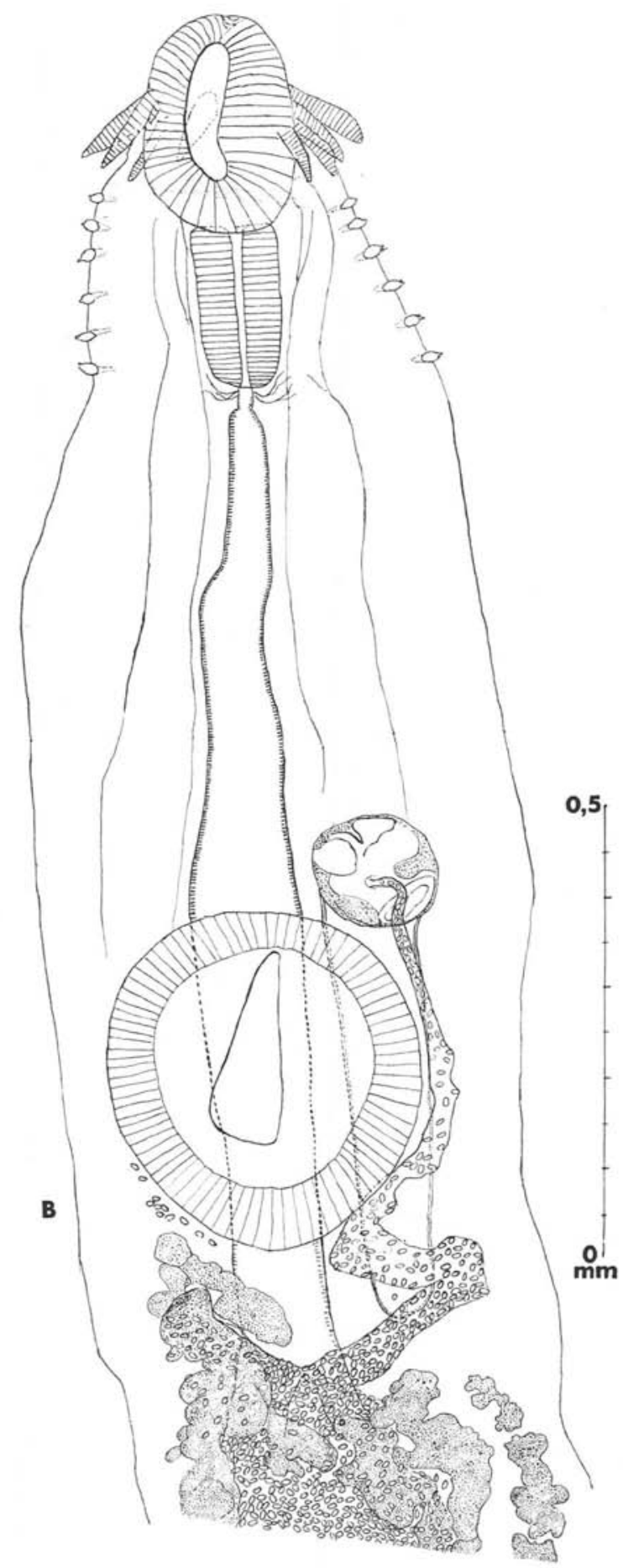

FIG. B. - Individu de même provenance. Partie antérieure, préovarienne 


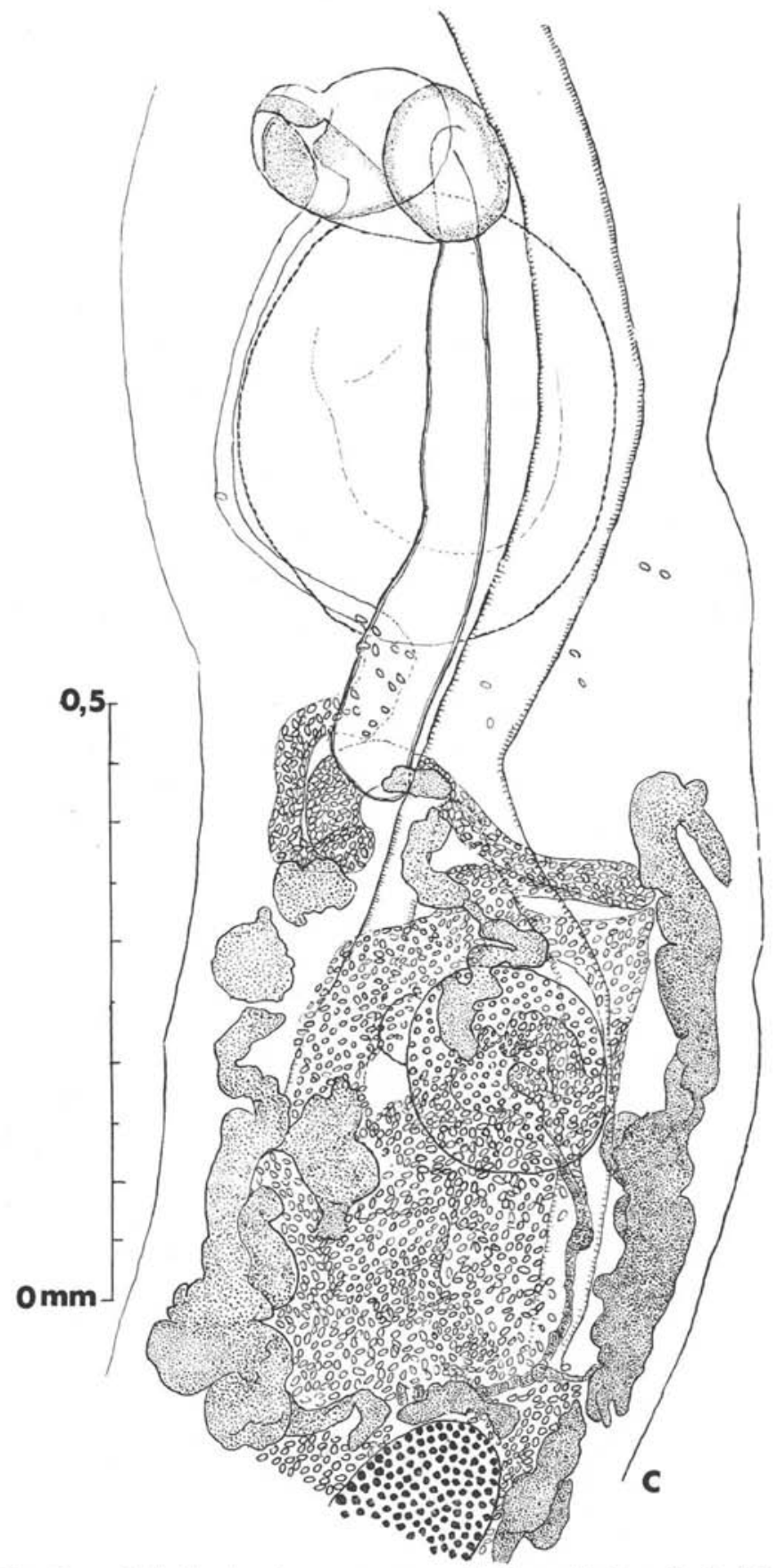

Fig. C. - Individu de même provenance. Partie acetabulo-prétesticulaire 
laire. La partie antérieure, dilatée, préacétabulaire, contient la pras prostatica, un cirre plusieurs fois replié sur lui-même et l'atrium génital.

Ovaire à contour circulaire, avec un diamètre de $0,17-0,20 \mathrm{~mm}$, situé un peu en arrière de la bifurcation intestinale. Canal de Laurer non observable, caché par des œufs. Utérus étendant ses sinuosités dans toute la partie du corps en arrière de la bifurcation intestinale, dorsalement et ventralement à l'ovaire et aux testicules. Plus antérieurement, il croise ventralement l'extrémité postérieure de la poche du cirre, suit le côté droit de celle-ci et le bord droit de la ventouse ventrale; atteignant sous forme de métraterme, l'atrium génital.

Les œufs, excessivement nombreux, mesurent de $20 \times 12,5 \mu$ à $22 \times 14 \mu$.

Les vitellogènes se présentent comme des masses irrégulières; ils s'étendent antérieurement presque jusqu'à la ventouse ventrale et postérieurement jusqu'à la mi-longueur du testicule postérieur. Il y a des follicules d'un côté qui sont très rapprochés de ceux de l'autre côté, mais ils ne paraissent pas confluents; ils sont ventraux par rapport à l'ovaire et dorsaux par rapport à l'intestin. Le vitelloducte transverse n'est pas observable.

La vessie excrétrice, masquée par les œufs n'a pas été bien observée; je la suppose conforme aux descriptions données par S. W. Hopkins (1940, p. 282-283, fig. 3-6) et par H.W. Manter (1947, p. 323, fig. 88) pour le Tergestia qu'ils ont rapporté à pectinata (Linton), mais que je considère comme une espèce différente de celle de Linton.

Discussion : Pour des Tergestia sous leur forme adulte (1) chez des Téléostéens, 10 noms spécifiques ont été créés jusqu'à présent (1970). Ce sont, par ordre chronologique :

Dimension des œufs $(\mu)$

1. T. laticollis (Rudolphi, 1819) M. Stos- $21-23 \times 15$ (T. Odhner, 1911, p. 112). sich 1899. Type du genre.

27-24 $\times 15-13$ (P. V. Wlassenko, 1932, p. 102).

23-26 $\times 18$ (S. Yamaguti, 1938, p. 97).

$24 \times 15-18$ in vivo; $21-30 \times 16-18$ montés

(S. Yamaguti, 1951, p. 270).

25-30 $\times 16-21$ (S. Yamaguti, 1970, p. 39).

22-26 $\times 12-17$ (H. W. Manter, 1940, p. 409).

(Océan Pacifique).

$25-29 \times 17-20$ (H. W. Manter, 1940, p. 409)

(Océan Atlantique).

2. T. acanthocephala (M. Stossich, 1887) ?

M. Stossich, 1899.

3. T. priacanthi (G. A. Mac Callum, 1917).

(1) Les descripteurs n'ont pas toujours indigué les dimensions des œufs. Par exemple, P. Olsson (1868, p. 29, pl. IV, fig. 76-78), pour un spécimen unique, récolté dans l'intestin de Trachurus trachurus (L.) à Bergen et qu'il désigna sous le nom inexact de "Distoma Polonii Molin », alors qu'il s'agissait de Tergestia laticolle (Rud.), ainsi cue le reconnut F. S. Monticelli (1890, p. 423). Ce spécimen unique fut examiné par T. Odhner (1911, p. 113) qui confirma l'appartenance à laticolle et, à cette occasion, indiqua que le vrai Distoma Polonii Molin - non Olsson - doit s'appeler Pharyngora polonii (Molin 1859) T. Odhner 1911. 
4. T. pectinata (Edwin Linton, 1905) $25 \times 14$ (E. Linton, 1905, p. 366).

non, H. W. Manter, 1940.

22-15 $\times 18-17$ (E. Linton, 1905, p. 389).

$24 \times 17$ et $20 \times 14$ (E. Linton, 1910, p. 41).

$24 \times 18$ (E. Linton, 1940, p. 44).

5. T. acanthogobii, S. Yamaguti, 1938.

21-27 $\times 14-18$ (S. Yamaguti, 1938, p. 98).

6. T. acuta, H. W. Manter, 1947.

$17-19 \times 9-10$ (H.W. Manter, 1947, p. 323).

7. T. agnostomi, H. W. Manter.

? (immature)

8. T. clonacantha, H. W. Manter, 1963.

15-18 × 9-12 (H.W. Manter, 1963, p. 447).

9. T. pauca, T. de Freitas et Anna Kohn.

10. T. Kuhliae, S. Yamaguti, 1970.

$22 \times 15-17$ (T. de Freitas et Anna Kohn, 1965 , p. 293).

A ces dix noms spécifiques, j'ajoute :

11. $T$. manteri, nom. nov. $=$ pectinata $20 \times 13$ (S. H. Hopkins, 1940, p. 283).

sensu, Manter et Hopkins, non Linton. $17-20 \times 10-15$ (H. W. Manter, 1947, p. 323).

Notre espèce parasite de Pomatomus est à comparer à celles ci-dessus mentionnées. Elle a comme caractères:

a) Ventouse ventrale environ deux fois plus grande que l'orale, ou un peu plus de deux fois ;

b) Pharynx environ deux fois plus long que large;

c) Long œsophage, se divisant en deux branches intestinales en arrière de la ventouse ventrale ;

d) Poche du cirre très longue, dépassant postérieurement la ventouse ventrale.

Ces caractères ne sont réunis chez aucune des espèces ci-dessus mentionnées. Une d'elles, cependant, est très voisine, c'est pectinata sensu Hopkins 1940 et sensu Manter 1947, non Linton 1905.

Notre espèce en diffère : $1^{\circ}$ par la présence, au niveau du pharynx, de protubérances sacciformes terminées par deux courts filaments. Ces protubérances ont une disposition très voisine de celles observées par Edwin Linton (1905, pl. XVIII, fig. 201) pour Tergestia pectinata (Linton) parasite de Bairdiella chrysura (Lacepède 1802) (Sciaenidae) à Beaufort (Caroline du Nord) et par H. W. Manter (1963, p. 444-445, fig. 5-6) pour T. clonacantha Manter d'un Hemiramphus sp. (Hemiramphidae) des Fiji (2).

$2^{\circ}$ par un beaucoup plus court œsophage ;

$3^{\circ}$ par sa poche du cirre rectiligne et non sinueuse comme l'a figurée Manter (1947, fig. 85$)$;

(2) Ce n'est peut-être pas la même espèce qui a été figurée sous le nom de $T$. clonacantha Manter, par M. Hafeezullah et Ather H. Siddiqui (1970, p. 939, fig. 13): les lobes céphaliques sont très différents et les protubérances collaires ont un emplacement un peu différent. 
Pour pectinata, Edwin Linton (1905, pl. XVIII fig. 200-201) figure la bifurcation intestinale nettement en avant de la ventouse ventrale, conformément à la description qu'il donne (ibid. p. 289) de l'espèce (3). Manter (1947, p. 404, fig. 85) figure la bifurcation au niveau du bord postérieur de la ventouse centrale, il n'est pas admissible que la bifurcation soit, chez la même espèce, tantôt en avant, tantôt en arrière de cette ventouse. Je considère donc le «pectinata» de Manter comme une espèce différente du pectinata de Linton et je propose de la nommer Tergestia manteri, nomen novum.

Le Tergestia décrit sous le nom «Tergestia pectinata (Linton 1905) Manter 1940 » par F. Sogandarès-Bernal et R. F. Hutton (1959, p. 64, fig. 10-11), parasite de Caranx chrysos (Mitchill), de Floride, a la bifurcation œsophagienne au niveau du bord antérieur de l'acetabulum et non pas en arrière, il appartient donc au sous-genre Theledera Linton, mais non pas au sous-genre Tergestia sensu Hopkins (1940) et Manter (1947). (Dimensions des œufs non indiquées).

F. Songandarès-Bernal (1959, p. 101-102) n'ayant donné ni description, ni figure du Tergestia qu'il désigne sous le nom «Tergestia pectinata (Linton 1905), Manter 1940 » et trouvé chez plusieurs espèces de poissons (Caranx, Selar), à Bimini (Floride), on ne peut savoir si, chez les spécimens qu'il a récoltés, la bifurcation de l'œsophage était en avant de l'acetabulum (sous-genre Theledera Linton 1910) ou en arrière (sousgenre Tergestia Stossich 1899).

Le Tergestia parasite de Chloroscombrus chrysurus (L. 1766) de Porto-Rico, figuré sous le nom $T$. pectinata (Linton 1905) Manter 1940, par A. Siddiqi et R. M. Cable (1960, p. 284-285, pl. VII, fig. 43) n'a pas la bifurcation de l'œsophage en avant de la ventouse ventrale comme l'a décrit et figuré Linton, ce n'est donc pas pectinata Linton, mais manteri mihi.

L'espèce parasite de Caranx chrysos (Mitchill) de Floride, décrite par R. M. Overstreet (1969, p. 123) sous le nom «Tergestia pectinata (Linton, 1905) Manter 1940 ne peut pas être à la fois l'espèce décrite sous ce nom spécifique par Linton et celle décrite sous le même nom spécifique par Manter: celle de Linton a l'œsophage se divisant en avant de l'acetabulum (s.g. Theledera) celle de Manter a l'œsophage se divisant en arrière de l'acetabulum. Pour les œufs, Overstreet a mesuré 19-23 $\times 12-14 \mu$.

En ce qui concerne Tergestia sp. Overstreet (1969, p. 123-124, fig. 2) de Selene vomer (L. 1758), de Floride, on peut admettre, d'après la figure que la bifurcation de l'œsophage n'est pas préacétabulaire et qu'il ne s'agit pas du sous-genre Theledera Linton 1910. Les œufs mesurent 16-23 × 11-16 $\mu$.

En ce qui concerne l'espèce mauritanienne parasite de Pomatomus, je lui donne le nom de Tergestia mauritanica $\mathrm{n}$. sp.

(3) Genre Theledera Edwin Linton 1910. La bifurcation préacetabulaire existe chez d'autres espèces; chez acanthocephala (M. Stossich 1887, p. 94, pl. X, fig. 40), chez priacantha (G. A. Mac Callum 1917, p. 70, fig. 34) (genre Cithara, G. A. Mac Callum 1917), chez kuhlia (S. Yamaguti 1970, p. 38, pl. IX, fig. 48). Elle est aussi figurée par F.S. Monticelli (1890, pl. XXII, fig. 1) pour laticollis, mais c'est une erreur évidente, d'après T. Odhner (1911, p. 110, note 19). 


\section{Tergestia sp.}

\section{Matériel de la collection :}

$1^{\circ} 6$ individus, du tube digestif de Lichia vadigo (A. Risso 1810) $=$ Campogramma lirio $\mathrm{R}$. Ph. Dollfus 1955 (Carangidae). $1^{\text {re }}$ et $2^{\circ}$ croisières du S. S. Boula-Matari.

$2^{\circ} 5$ individus du tube digestif d'un poisson non précisé, mais qui était probablement aussi Lichia vadigo (A. Risso). $1^{\text {re }}$ croisière du S. S. Boula-Matari 22-12-1922.

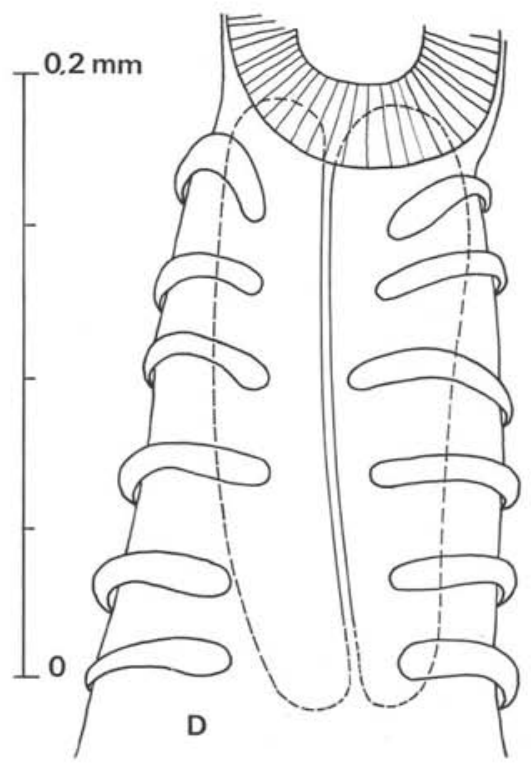

Fig. D. - Région pharyngienne de Tergestia sp. de l'intestin de Lichia vadigo (Risso 1810). Parages de Port-Etienne (Mauritanie). 22-12-1922

Description : Longueur du corps $2 \mathrm{~mm}$, largeur $0,3 \mathrm{~mm}$.

Appendices céphaliques de la ventouse orale plutôt courts $(87 \mu)$. Plis cuticulaires de la face ventrale de la région pharyngienne plutôt larges (env. 12,5 $\mu$ ), interrompus sur la ligne médiane (disposition existant aussi chez priacanthi, G. A. Mac Callum, 1917, fig. 34), se terminant par une protubérance marginale en forme d'arceau.

Ventouse orale $200 \times 185 \mu$; ventouse ventrale $310 \times 250 \mu$, presque deux fois plus grande que l'orale. Pharynx deux fois plus long que large $(196 \times 100 \mu$ et $205 \times 83 \mu)$.

Bifurcation intestinale postacétabulaire.

Vitellogènes débutant au niveau du bord postérieur de l'acétabulum et dépassant postérieurement le milieu du testicule postérieur, sans atteindre l'extrémité postérieure du corps.

Eufs, $22 \times 12,2-14,6 \mu$, excessivement nombreux, cachant presque complètement les organes. 


\section{Discussion.}

Le fait que la bifurcation intestinale est postacetabulaire élimine l'attribution à acanthocephala, priacanthi, pectinata Linton (non Manter) et à kuhlia Yamaguti.

Le fait que la ventouse ventrale est presque 2 fois plus grande que l'orale élimine les espèces où la différence est moindre : laticollis, agnostomi, cloacantha, acanthogobii, priacanthi.

Il ne s'agit pas de mauritanica ni de manteri, les protubérances marginales de la région pharyngienne étant différentes.

Notre matériel étant médiocrement conservé, nous préférons ne pas proposer d'attribution spécifique.

$$
\text { * }
$$

Pour comparaison, j'aurais voulu donner une bonne figure du type du genre ; malheureusement, dans ma collection, je possède seulement des individus très contractés, à peine reconnaissables. Je figure (fig. E) l'un d'entre eux, que j'ai récolté dans l'intestin d'un Trachurus trachurus (L.) des parages d'Agadir (Maroc) (mission du S. S. "Vanneau »s $S^{\text {tion }}$ LXXXII, 31-8-1925). Ce spécimen mesure $1,10 \times 0,47 \mathrm{~mm}$; il est pourvu de 13 appendices céphaliques. Les œufs, excessivement nombreux $(24,4 \times 17,1$; $24,4 \times 19,5 ; 29,9 \times 17,1 \mu$ ) cachent une grande partie des organes. Les yeux cercariens sont encore présents, malgré la maturité génitale.

Un Tergestia parasite de Euthynnus alletteratus (Rafinesque), Selene vomer (L.), Anchoviella epsetus (Bonnaterre) de Porto-Rico, a été rapporté à laticollis (Rud. 1819) par A. Siddiqi et R. M. Cable (1960, p. 284, pl. VI, fig. 41, pl. VII, fig. 42a-B). Un individu provenant de Gerres cinereus (Walbaum) avait la même formule de flammes que celle décrite par Hopkins (1940) et 14 appendices céphaliques; un autre, provenant de Selene vomer (L.), avait une formule de flammes un peu différente et 13 appendices céphaliques. Siddiqi et Cable suggèrent qu'il pourrait s'agir d'une espèce distincte.

Je doute que le laticollis figuré par Siddiqi et Cable soit le même que celui de Rudolphi, de Stossich et d'Odhner : il a la ventouse orale proportionnellement plus grande et les vitellogènes beaucoup moins étendus.

\section{Bibliographie}

Une partie seulement des publications où il est question de Tergestia est citée ci-dessous.

ANGel (L.-Madeline), 1960. - Cercaria haswelli Dollfus, 1927; a re-examination of Haswell's material, with discussion of the genus Tergestia. Libro Homenaje al Dr. Eduardo Caballero y Caballero, Mexico, 1960, p. 75-86, fig. 1-10.

Dollfus (Robert-Ph.), 1964. - Metacercaria: Proctoeces progeneticus (Trematoda Digenea) chez une Gibbula (Gastropoda Prosobranchiata) de la côte atlantique du Maroc. Observations sur la famille Fellodistomatidae. Ann. Parasitol. hum. et comp., t. XXXIX, $\mathrm{n}^{\circ} 6,1964$, p. 755-774, fig. 1-4. 


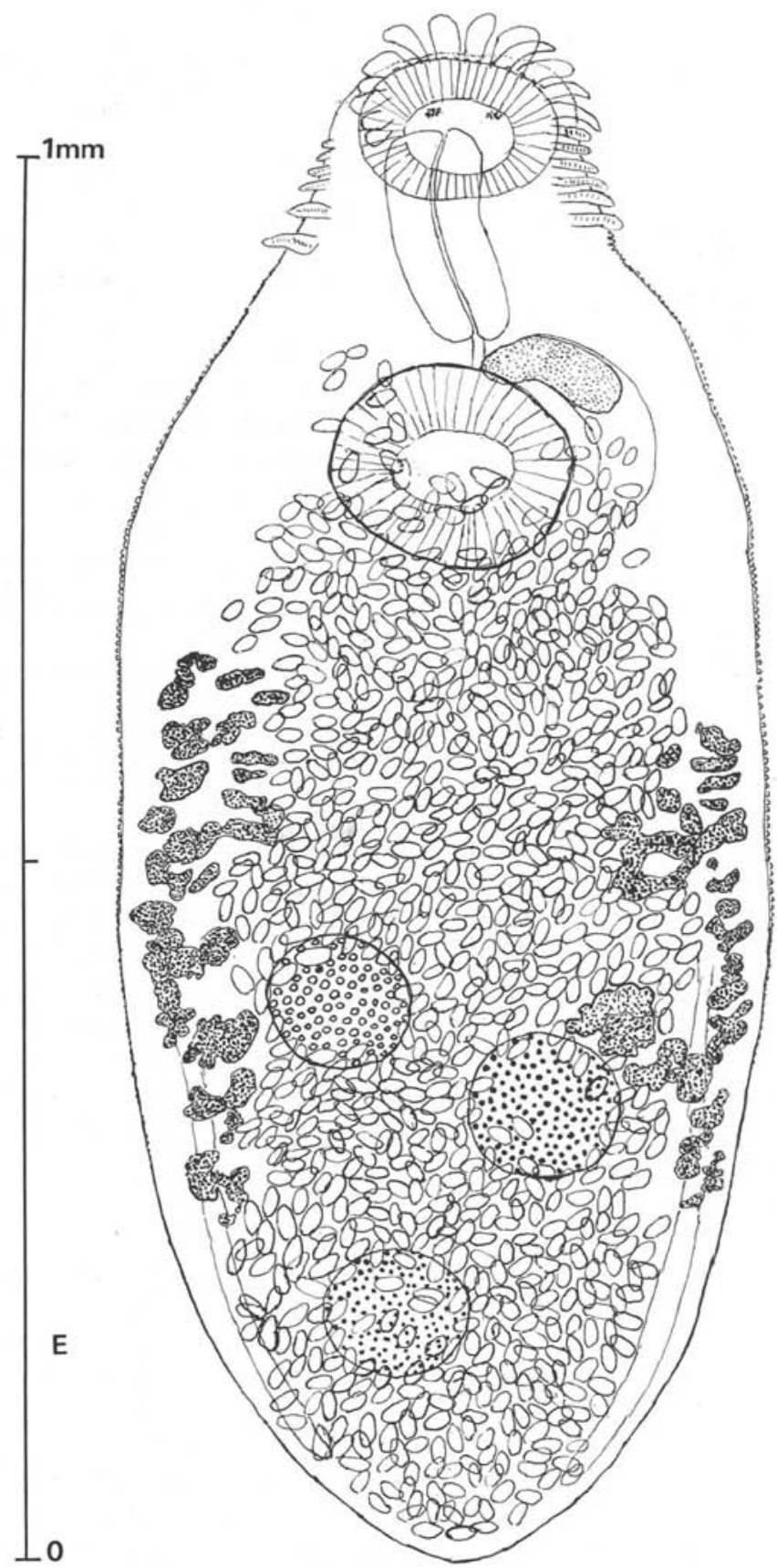

FIG. E. - Tergestia laticollis (Rudolphi 1819) Stossich 1899. Individu très contracté, de l'intestin de Trachurus trachurus (L. 1758). Agadir (Maroc). 31-1-1925 
Freitas (J. F. Teixeira de -) et Kohn (Anna), 1965. - Nova especie do genero Tergestia Stossich, 1899 (Trematoda, Fellodistomidae). Mem. Inst. Oswaldo Cruz, t. LXIII, fasc. unique, 1965, p. 291-297, fig. 1-2.

Hafeezullah (M). et Sjddier (Ather-H.), '1970. — Digenetic Trematodes of marine fishes of India. Part. II. Fellodistomatidae. Journ. Parasitology, vol. LVI, ${ }^{\circ} 5$, oct. 1970, p. 932-940, fig. 1-13.

Hopkins (Sewell H.), 1940. - The excretory systems of Tergestia Stossich, 1899 and Cornucopia adunca (Linton, 1905) (Trematoda). Transact. of the american microscop. Soc., vol. LIX, $\mathrm{n}^{\circ} 3$, July 1940 , p. 281-284, fig. 1-4.

Linton (Edwin), 1905. - Parasites of Fishes of Beaufort, North Carolina. Bull. Bureau of Fisheries, vol. XXIV (1904), 1905, p. 321-428, pl. I-XXXIV, fig. 1-249.

-, 1910. - Helminth Fauna of the Dry Tortugas. Papers from the Tortugas, Laboratory of the Carnegie Institution of Washington, vol. IV, $\mathrm{n}^{\circ} 133,16-12-1910$, p. 11-98, pl. I-XVIII, fig. 1-241.

—, 1940. - Trematodes from Fishes mainly from the Woods Hole Region Massachusetts. Proc. Un. St. national Museum, $\mathrm{n}^{\circ}$ 3078, vol. LXXXVIII, p. 1-72, pl. I-XXVI, fig. 1-351.

Mac Callum (G. A.), 1917. - Some news forms of parasitic worms. Zoopathologia, New York, vol. I, $\mathrm{n}^{\circ}$ 2, July 1917 , p. 43-75, fig. 1-36.

Manter (Harold W.), 1940. - Digenetic Trematodes of fishes from the Galapagos Islands and the neighboring Pacific. Allan Hancock Pacific Expeditions, vol. 2, $\mathrm{n}^{\circ} 14$, 27-3-1940, p. 127-497, pl. XLII-L, fig. 1-136.

-, 1947. - The Digenetic Trematodes of marine fishes of Tortugas, Florida. Amer. Midland Naturalist, vol. XXXVIII, $\mathrm{n}^{\circ}$ 2, sept. 1947, p. 257-416, fig. 1-152.

-, 1954. - Some Digenetic Trematodes from fishes of New Zealand. Transact. royal Soc. New Zealand, vol. LXXXII, part 2, sept. 1954, p. 475-568, fig. 1-89.

-, 1963. - Studies on Digenetic Trematodes of Fishes of Fiji. III. Families Acanthocolpidae, Fellodistomatidae, and Cryptogonimidae. Journ. Parasitology, vol. XLIX, $\mathrm{n}^{\circ}$ 3, June 1963, p. 443-450, pl. I, fig. 1-9, pl. II, fig. 10-16.

Monticelli (Francesco Saverio), 1890. - Elenco degli elminti studiati a Wimereux nella primavera del 1889. Bull. scient. France-Belgique, t. XII, $2^{\circ}$ partie, 21-7-1890, p. 417-444, pl. XXII, fig. 1-27.

ODHNER (Teodor), 1911. - Zum natürlichen System der digenen Trematoden. III. Zoolog. Anzeiger, Bd, XXXVIII, Nr 4, 8-8-1911, p. 97-117, fig. 1-8.

OLsson (Peter), 1868. - Entozoa, iakttagna hos Skandinaviska hafsfiskar. Lunds Univers. Ărsskrift, r. IV (1868-1869), p. 1-64, pl. III-V, fig. 52-108.

Overstreet (Robin M.), 1969. - Digenetic Trematodes of marine teleost fishes from Biscayne Bay, Florida. Tulane Studies in Zoology and Botany, vol. XV, $\mathrm{n}^{\circ} 4$, June 1969, p. 119-170, fig. 1-37.

Siddier (Ather H.) et CABLE (Raymond-M.), 1960. - Digenetic Trematodes of marine fishes of Puerto Rico. Scientific Survey of Porto Rico and Virgin Islands, vol. XVII, part. 3, 15-7-1960, p. 255-369, pl. I-XXI, fig. 1-139.

Sogandares-Bernal (Franklin), 1959. - Digenetic Trematodes of marine Fishes from the Gulf of Panama and Bimini, British West Indies. Tulane Studies in Zoology, vol. VII, $\mathrm{n}^{\circ} 3,24-8-1959$, p. 69-117, fig. 1-62. 
- et Hutron (Robert), 1959. - Studies on helminth parasites of the coast of Florida. I. Digenetic Trematodes of marine Fishes from Tampa and Boca Ciega Bays with descriptions of two new species. Bull. marine Science of the Gulf and Caribbean, vol. IX, $\mathrm{n}^{\circ} 1$, March 1959, p. 53-68, fig. 1-17.

Srossich (Michele), 1887. - Brani di elmintologia tergestina. Serie quarto. Bolletino $d$. Societa adriatica di scienze naturali Trieste, vol. IX, 1887, p. 90-96, pl. X, fig. 38-41.

-, 1899. - Le sezione degli Echinostomi. Bolletino d. Societa adriatica di scienze naturali Trieste, vol. XIX, 1899, p. 11-16.

Yamaguti (Satyu), 1934. - Studies on the Helminth Fauna of Japan. Part. 2. Trematodes of Fishes I. Japanese Journ. Zoology, vol. V, $\mathrm{n}^{\circ}$ 3, 31-3-1934, p. 249-541, fig. 1-145.

—, 1938. - Studies on the Helminth Fauna of Japan. Part 21. Trematodes of Fishes, IV. Kyoto, April 10, 1938, p. 1-139, fig. 1-83, 1 pl. fig. 1-9.

-, 1951. - Studies on the Helminth Fauna of Japan. Part 44. Trematodes of Fishes, IX. Arbeiten medizinischen Fakultät Okayama, Bd VII, Heft 4, August 1951, p. 247 282, pl. I-V, fig. $1-16$.

—, 1970. - Digenetic Trematodes of Hawaiian Fishes. Keigaku Publishing Co., Tokyo, oct. 1970, III + 1-436 p., pl. I-LXXXVIII, fig. 1-341.

WlassenKo (P.), 1931. - Zur Helminthofauna der Schwarzmeer Fische. Trav. Station biologique à Karadagh, Livre IV (1931), 1932, p. 88-136, fig. 1-26. 